\title{
ANGIOGENESIS
}

\section{Blunting the spikes}

DOI:

10.1038/nrc2018

URLs
The role of circulating endothelial progenitors (CEPs; bone-marrow derived endothelial progenitor cells that enter the circulation and migrate to sites of angiogenesis) in tumours has been controversial. Their contribution to tumour endothelium has been estimated to be as low as $5 \%$ or less in many studies of tumours from untreated hosts. However, Robert Kerbel and colleagues have now shown that if CEPs are acutely mobilized in tumour-bearing mice using vascular disrupting agents (VDAs), treatment with anti-angiogenic agents disrupts this spike in CEP numbers, and thereby reduces tumour cell growth at the viable tumour periphery that remains after VDA treatments. The use of VDAs combined with anti-angiogenic agents might therefore be an efficacious combination to treat solid tumours.

VDAs cause the acute occlusion of existing blood vessels and rapid tumour necrosis - unlike antiangiogenic agents that mainly inhibit new blood vessel formation. The authors proposed that the rapid mobilization of CEPs as a consequence of treatment with VDAs might contribute to the rapid regrowth that is seen at the tumour periphery after VDA treatment. First, the authors showed that the treatment of non-tumour-bearing mice with the VDA combretastine-A4 phosphate or its more potent derivative OXi-4503 did cause a CEP spike of about three times normal levels. If the anti-angiogenic agent DC101 was injected into tumour-bearing mice 24 hours before giving OXi-4503, the size of the viable tumour rim was reduced threefold compared with that seen in mice treated with OXi-4503 alone. In addition, the combination of the VDA and anti-angiogenic agent led to markedly decreased perfusion, increased hypoxia and necrosis of the tumours.

So, does the VDA-induced spike in CEPs lead to preferential homing of the cells to the viable tumour rim? To investigate this, Kerbel and colleagues used lethally irradiated mice transplanted with green fluorescent protein $\left(\mathrm{GFP}^{+}\right)$bone marrow cells before allowing syngeneic Lewis lung carcinomas to establish. Although untreated and DC101-treated mice showed only some modest homing of the bone marrow cells to the tumour rim, mice treated with OXi-4503 alone showed greater homing and incorporation of some of the $\mathrm{GFP}^{+}$ cells into the peripheral tumou vasculature. Mice treated with both drugs had a reduced number of $\mathrm{GFP}^{+}$cells at the tumour periphery. If mice that are incapable of mobilizing CEPs $\left(\mathrm{Id}^{\mathrm{mut}}\right.$ mice) are treated with OXi-4503 no CEP spike is seen.

When these mice bear Lewis lung tumours and are treated with OXi-4503, the viable tumour rim and perfusion are reduced, confirming the significant role of therapy-induced mobilization and tumour-homing of CEPs in tumour regrowth.

The authors conclude that CEPs are a key contributor to the growth of the viable tumour rim after treatment with VDAs, and suggest that treatment with cytotoxic chemotherapy drugs at maximum tolerated doses might have the same effect. If so, the combination of an anti-angiogenic drug given with chemotherapy might be one way the anti-tumour efficacy of chemotherapy can be improved.

Ezzie Hutchinson

ORIGINAL RESEARCH PAPER Shaked, Y. et al.

Therapy-induced acute recruitment of circulating endothelial progenitor cells to tumors. Science 313, 1785-1787 (2006)

FURTHER READING Bertolini, F., Shaked, Y. Mancuso, P. \& Kerbel, R. S. The multifaceted circulating endothelial cell in cancer: towards marker and target identification. Nature Rev. Cancer 5 October 2006 (doi:10.1038/nrc1971) 